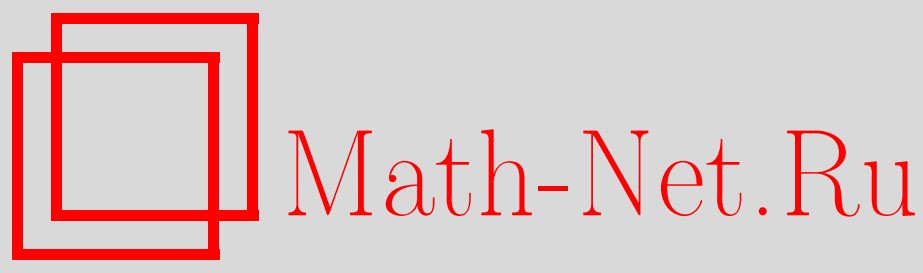

С. Буаррудж, Тернарные инвариантные дифференциальные операторы, действующие на пространстве взвешенных плотностей, ТМФ, 2009, том 158, номер 2, 165180

DOI: https://doi.org/10.4213/tmf6307

Использование Общероссийского математического портала Math-Net.Ru подразумевает, что вы прочитали и согласны с пользовательским соглашением http://www.mathnet.ru/rus/agreement

Параметры загрузки:

IP : 3.93 .64 .190

26 апреля 2023 г., $11: 23: 26$

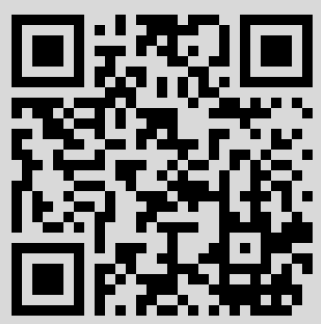




\title{
ТЕРНАРНЫЕ ИНВАРИАНТНЫЕ ДИФФЕРЕНЦИАЛЬНЫЕ ОПЕРАТОРЫ, ДЕЙСТВУЮЩИЕ НА ПРОСТРАНСТВЕ ВЗВЕШЕННЫХ ПЛОТНОСТЕЙ
}

\begin{abstract}
Приведена классификация тернарных дифференциальных операторов на $n$-мерных многообразиях. Эти операторы действуют на пространствах взвешенных плотностей и инвариантны относительно алгебры Ли векторных полей. При $n=1$ некоторые из них можно выразить в терминах внешнего дифференциала де Рама, скобки Пуассона, оператора Грозмана и антисимметричных операторов Фейгина-Фукса; четыре (с точностью до дуализаций и перестановок) оператора являются новыми. При $n>1$ приведен список многомерных конформных трансвекторов, т.е. операторов, действующих на пространстве взвешенных плотностей и инвариантных относительно конформной алгебры Ли $\mathfrak{o}(p+1, q+1)$, где $p+q=n$. Все эти конформно-инвариантные операторы, за исключением скалярного, не инвариантны относительно всей алгебры Ли векторных полей.
\end{abstract}

Ключевые слова: инвариантные операторы, трансвекторы, тензор плотностей, конформные структуры.

\section{1. ВВЕДЕНИЕ}

Пусть $M^{n}$ - многообразие, а $\mathfrak{v e c t}(M)$ - алгебра Ли векторных полей на $M$ с коэффициентами, свойства которых мы укажем позднее (гладкие, полиномиальные и т.д. коэффициенты). Пусть $T(V)-\mathfrak{v e c t}(M)$-модуль тензорных полей типа $V$ для представления $\rho: G L(n) \rightarrow G L(V)$ (см. работы [1], [2] по поводу точного описания $\mathfrak{v e c t}(M)$-действия). Положим $\mathfrak{v e c t}(n):=\mathfrak{v} \mathfrak{e c t}\left(\mathbb{R}^{n}\right)$.

Все законы физики описываются операторами $D: T\left(V_{1}\right) \otimes \cdots \otimes T\left(V_{k}\right) \rightarrow T(W)$, инвариантными относительно действия алгебры $\mathfrak{v e c t}(M)$ или какой-то ее подалгебры; этим и определяется важность таких операторов [3]. Задача классификации инвариантных операторов сформулирована Вебленом в 1928 г. на Международном конгрессе математиков в Болонье [4]. С тех пор были построены обобщения многих

${ }^{*}$ Department of Mathematical Sciences, U.A.E. University, Al-Ain, U.A.E.

E-mail: bouarroudj.sofiane@uaeu.ac.ae 
операторов, известных ранее лишь для частных случаев, а также найдено несколько новых операторов.

В настоящей работе приводится список тернарных $\mathfrak{v e c t}(1)$-инвариантных дифференциальных операторов, действующих на тензорных полях на $\mathbb{R}$. Ясно, что пространства $T(V)$ на одномерных многообразиях имеют вид $\mathcal{F}_{\lambda}=\left\{f d x^{\lambda} \mid f \in \mathbb{R}[x]\right\}$, т.е. являются пространствами взвешенных плотностей веса $\lambda \in \mathbb{R}$.

В унарном случае в работах [4]-[6] было независимо и различными методами доказано, что имеется только один инвариантный дифференциальный оператор внешний дифференциал. В частности, при $n=1$ имеем

$$
d: \mathcal{F}_{0} \rightarrow \mathcal{F}_{1}, \quad \varphi \mapsto \varphi^{\prime} d x
$$

На пространстве функций с компактным носителем или в случае, когда $M$ компактно, имеется также недифференциальный оператор - интеграл:

$$
\int: \mathcal{F}_{1} \rightarrow \mathbb{R} \subset \mathcal{F}_{0}, \quad \varphi d x \mapsto \int \varphi d x
$$

Все рассматриваемые ниже функции являются полиномиальными, а операторы дифференциальными. Как описывать недифференциальные операторы, не ясно, и приведенный Кирилловым пример недифференциального оператора, отличного от интеграла (см. [1], [4]), делает ситуацию лишь более загадочной.

Заметим, что, во-первых, следующий скалярный оператор является инвариантным при любой "арности" $k$ :

$$
\begin{aligned}
\mathcal{F}_{\lambda_{1}} \otimes \cdots \otimes \mathcal{F}_{\lambda_{k}} & \rightarrow \mathcal{F}_{\lambda_{1}+\cdots+\lambda_{k}}, \\
\varphi_{1}(d x)^{\lambda_{1}} \otimes \cdots \otimes \varphi_{k}(d x)^{\lambda_{k}} & \mapsto\left(\prod \varphi_{i}\right)(d x)^{\lambda_{1}+\cdots+\lambda_{k}}
\end{aligned}
$$

во-вторых, каждый инвариантный дифференциальный оператор имеет постоянные коэффициенты [4]. В бинарном случае классификация (для любого $n$ ) была дана Грозманом [7]. В частности, при $n=1$ имеем следующие операторы.

Операторы первого порядка:

$$
\begin{array}{ll}
\mathcal{F}_{0} \otimes \mathcal{F}_{0} \rightarrow \mathcal{F}_{1}, & \varphi \otimes \psi \mapsto\left(a \varphi^{\prime} \psi+b \varphi \psi^{\prime}\right) d x \quad \text { при любых } \quad a, b \in \mathbb{R}, \\
\mathcal{F}_{\lambda} \otimes \mathcal{F}_{\mu} \rightarrow \mathcal{F}_{\lambda+\mu+1}, & \varphi(d x)^{\lambda} \otimes \psi(d x)^{\mu} \mapsto\left(-\lambda \varphi \psi^{\prime}+\mu \varphi^{\prime} \psi\right)(d x)^{\lambda+\mu+1} .
\end{array}
$$

Заметим, что второй из этих операторов является скобкой Пуассона в координатах $x$ и $p:=d x$. Ниже этот оператор будет обозначаться как $\left\{\varphi(d x)^{\lambda}, \psi(d x)^{\mu}\right\}$.

Операторы второго порядка:

$$
\begin{array}{ll}
\mathcal{F}_{0} \otimes \mathcal{F}_{\mu} \rightarrow \mathcal{F}_{\mu+2}, & \varphi \otimes \psi(d x)^{\mu} \mapsto\left(-\varphi^{\prime} \psi^{\prime}+\mu \varphi^{\prime \prime} \psi\right)(d x)^{\mu+2}, \\
\mathcal{F}_{\lambda} \otimes \mathcal{F}_{0} \rightarrow \mathcal{F}_{\lambda+2}, & \varphi(d x)^{\lambda} \otimes \psi \mapsto\left(-\lambda \varphi \psi^{\prime \prime}+\varphi^{\prime} \psi^{\prime}\right)(d x)^{\lambda+2}, \\
\mathcal{F}_{\lambda} \otimes \mathcal{F}_{-\lambda-1} \rightarrow \mathcal{F}_{1}, & \varphi(d x)^{\lambda} \otimes \psi(d x)^{-\lambda-1} \mapsto \\
& \mapsto\left(-\lambda \varphi \psi^{\prime \prime}-(2 \lambda+1) \varphi^{\prime} \psi^{\prime}-(\lambda+1) \varphi^{\prime \prime} \psi\right) d x .
\end{array}
$$


Операторы третьего порядка:

$$
\begin{array}{cl}
\mathcal{F}_{0} \otimes \mathcal{F}_{0} \rightarrow \mathcal{F}_{3}, & \varphi \otimes \psi \mapsto\left(\varphi^{\prime} \psi^{\prime \prime}-\varphi^{\prime \prime} \psi^{\prime}\right)(d x)^{3}, \\
\mathcal{F}_{0} \otimes \mathcal{F}_{-2} \rightarrow \mathcal{F}_{1}, & \varphi \otimes \psi(d x)^{-2} \mapsto\left(\varphi^{\prime} \psi^{\prime \prime}+3 \varphi^{\prime \prime} \psi^{\prime}+2 \varphi^{\prime \prime \prime} \psi\right) d x, \\
\mathcal{F}_{-2} \otimes \mathcal{F}_{0} \rightarrow \mathcal{F}_{1}, & \varphi(d x)^{-2} \otimes \psi \mapsto\left(\varphi^{\prime \prime} \psi^{\prime}+3 \varphi^{\prime} \psi^{\prime \prime}+2 \varphi \psi^{\prime \prime \prime}\right) d x, \\
\mathrm{Gz}: \mathcal{F}_{-2 / 3} \otimes \mathcal{F}_{-2 / 3} \rightarrow \mathcal{F}_{5 / 3}, & \varphi(d x)^{-2 / 3} \otimes \psi(d x)^{-2 / 3} \mapsto \\
& \mapsto\left(2 \varphi^{\prime \prime \prime} \psi+3 \varphi^{\prime \prime} \psi^{\prime}-3 \varphi^{\prime} \psi^{\prime \prime}-2 \varphi \psi^{\prime \prime \prime}\right)(d x)^{5 / 3} .
\end{array}
$$

Таким образом, при $n=1$ все инвариантные операторы имеют порядок не выше 3 и выражаются в виде композиции дифференциала де Рама и скобки Пуассона, за исключением оператора Грозмана Gz. В работе [8] этот оператор послужил отправной точкой классификации всех антисимметричных $k$-арных дифференциальных операторов.

Мы покажем, что при $n>1$ не существует $\mathfrak{v e c t}(n)$-инвариантных операторов, действующих на пространстве взвешенных плотностей, кроме скалярного оператора (1.1). Можно, однако, интересоваться инвариантностью относительно некоторой подалгебры в $\mathfrak{v e c t}(n)$. Наибольший интерес представляют максимальные простые подалгебры. При $n=1$ имеется единственная максимальная простая подалгебра $\mathfrak{s l}(2) \simeq \mathfrak{o}(1,2)$. Инвариантные относительно нее операторы уже классифицированы: в унарном случае это операторы Бола [9], в бинарном случае - трансвекторь Гордона [10] (см. [11]-[17] по поводу их применения; об инвариантных операторах в тернарном случае см. [18]).

При $n>1$ можно рассмотреть многомерные аналоги трансвекторов, инвариантных относительно различных максимальных простых конечномерных подалгебр в $\mathfrak{v e c t}(n)$, например относительно $\mathfrak{s l}(n+1)$ или $\mathfrak{o}(p+1, q+1)$, где $n=p+q$ (ср. с работой [19]). Активно изучались $\mathfrak{o}(p+1, q+1)$-инвариантные операторы, иногда называемые конформно-инвариантными. В унарном случае все конформно-инвариантные операторы даются степенями лапласиана [20]-[22]

$$
\Delta_{g}^{k}: \mathcal{F}_{\lambda} \rightarrow \mathcal{F}_{\lambda+2 k / n}
$$

где $\Delta_{g}$ - лапласиан, связанный с псевдоримановой метрикой $g$ сигнатуры $(p, q)$. В работе [23] проведена классификация $\mathfrak{o}(p+1, q+1)$-инвариантных бинарных операторов и доказано, что для почти всех значений весов пространство конформно-инвариантных операторов одномерно.

Заметим, что $\mathfrak{v e c t}(1) \simeq \mathfrak{k}(1)$, поэтому естественно также расширить поиск трансвекторов "в контактном направлении", подобно работе [24], где были классифицированы билинейные дифференциальные операторы, действующие на пространстве взвешенных плотностей и инвариантные относительно симплектической алгебры Ли $\mathfrak{s p}(2 n+2) \subset \mathfrak{k}(2 n+1)$, самой естественной из максимальных простых подалгебр Ли.

Основные результаты. Приведена классификация всех $\mathfrak{v e c t}(n)$-инвариантных тернарных дифференциальных операторов, действующих на пространстве взвешенных плотностей. Некоторые из этих инвариантных операторов выражаются в терминах унарных и бинарных, некоторые обобщают антисимметричные инвариантные операторы Фейгина-Фукса; четыре (с точностью до дуализации и перестано- 
вок) инвариантых оператора являются новыми. Также приведена классификация всех конформно-инвариантных тернарных дифференциальных операторов, действующих на пространстве взвешенных плотностей. Прямые вычисления и повторяющиеся рассуждения опущены.

\section{2. ИНВАРИАНТНЫЕ ОПЕРАТОРЫ НА $\mathbb{R}$ ИЛИ $S^{1}$}

2.1. Антисимметричные инвариантные операторы Фейгина-Фукса. В работе [8] приведена классификация всех антисимметричных $k$-арных инвариантных дифференциальных операторов на пространстве взвешенных плотностей. Напомним, что список тернарных операторов из этой работы таков:

$$
\begin{array}{rlrl}
\Delta_{\lambda, 3}: & \wedge^{3} \mathcal{F}_{\lambda} \rightarrow \mathcal{F}_{3 \lambda+3}, & d \circ \Delta_{-1,3}: & \wedge^{3} \mathcal{F}_{-1} \rightarrow \mathcal{F}_{1}, \\
\Upsilon: & \wedge^{3} \mathcal{F}_{-5 / 4} \rightarrow \mathcal{F}_{9 / 4}, & \Delta_{1,3} \circ(d \otimes d \otimes d): & \wedge^{3} \mathcal{F}_{0} \rightarrow \mathcal{F}_{6}, \\
& \Theta_{ \pm}: \wedge^{3} \mathcal{F}_{\lambda} \rightarrow \mathcal{F}_{3 \lambda+5} & \text { при } \quad \lambda=-\frac{9 \pm \sqrt{21}}{12},
\end{array}
$$

где операторы $\Delta_{\lambda, 3}, \Upsilon, \Theta_{ \pm}$соответственно определяются следующими выражениями: $(\varphi, \psi, \chi)(d x)^{\lambda} \mapsto$

$$
\begin{aligned}
& \mapsto\left|\begin{array}{ccc}
\varphi & \psi & \chi \\
\varphi^{\prime} & \psi^{\prime} & \chi^{\prime} \\
\varphi^{\prime \prime} & \psi^{\prime \prime} & \chi^{\prime \prime}
\end{array}\right|(d x)^{3 \lambda+3}, \\
& \mapsto\left(\begin{array}{ccc}
\varphi & \psi & \chi \\
\varphi^{\prime} & \psi^{\prime} & \chi^{\prime} \\
\varphi^{(5)} & \psi^{(5)} & \chi^{(5)}
\end{array}\left|+\frac{5}{2}\right| \begin{array}{ccc}
\varphi & \psi & \chi \\
\varphi^{\prime \prime} & \psi^{\prime \prime} & \chi^{\prime \prime} \\
\varphi^{(4)} & \psi^{(4)} & \chi^{(4)}
\end{array}|+2| \begin{array}{ccc}
\varphi^{\prime} & \psi^{\prime} & \chi^{\prime} \\
\varphi^{\prime \prime} & \psi^{\prime \prime} & \chi^{\prime \prime} \\
\varphi^{(3)} & \psi^{(3)} & \chi^{(3)}
\end{array} \mid\right)(d x)^{9 / 4}, \\
& \mapsto\left(\begin{array}{ccc}
\varphi & \psi & \chi \\
\varphi^{\prime} & \psi^{\prime} & \chi^{\prime} \\
\varphi^{(4)} & \psi^{(4)} & \chi^{(4)}
\end{array}|+2(\mp \sqrt{21}-4)| \begin{array}{ccc}
\varphi & \psi & \chi \\
\varphi^{\prime \prime} & \psi^{\prime \prime} & \chi^{\prime \prime} \\
\varphi^{(3)} & \psi^{(3)} & \chi^{(3)}
\end{array} \mid\right)(d x)^{3 \lambda+5},
\end{aligned}
$$

где во второй строке $\lambda=-5 / 4$.

2.2. Список vect(1)-инвариантных тернарных инвариантных дифференциальных операторов. Напомним, что с любым оператором $A: \mathcal{F}_{\lambda} \otimes \mathcal{F}_{\gamma} \otimes \mathcal{F}_{\tau} \rightarrow \mathcal{F}_{\mu}$ можно связать следующие объекты.

Во-первых, три дуализации $(i=1,2,3)$ [1], [4], [25]

$$
A^{* i}: \mathcal{F}_{\alpha} \otimes \mathcal{F}_{\beta} \otimes \mathcal{F}_{\nu} \rightarrow \mathcal{F}_{\delta}
$$

где $(\alpha, \beta, \nu, \delta)=(1-\mu, \gamma, \tau, 1-\lambda)$ для $i=1$, или $(\alpha, \beta, \nu, \delta)=(\lambda, 1-\mu, \tau, 1-\gamma)$ для $i=2$, или $(\alpha, \beta, \nu, \delta)=(\lambda, \gamma, 1-\mu, 1-\tau)$ для $i=3$. Эти дуализации существуют в силу определения, согласно которому $\left(\mathcal{F}_{\alpha}\right)^{*}:=\mathcal{F}_{1-\alpha}$. Предположив, что одно из этих пространств состоит из функций с компактным носителем (это позволяет интегрировать), мы можем утверждать, что это определение правильнее, чем более естественное с первого взгляда $\left(\mathcal{F}_{\alpha}\right)^{*}=\mathcal{F}_{-\alpha}$.

Во-вторых, перестановки

$$
A^{\sigma}: \mathcal{F}_{\alpha} \otimes \mathcal{F}_{\beta} \otimes \mathcal{F}_{\nu} \rightarrow \mathcal{F}_{\mu}, \quad A^{\sigma}(a, b, c)=A\left(\sigma^{-1}(a, b, c)\right),
$$

где $(\alpha, \beta, \nu)=\sigma(\lambda, \gamma, \tau)$ для любого $\sigma \in S_{3}$. 
2.2.1. Порядки 1 и 2. Всюду в дальнейшем все $\epsilon_{j}-$ константы, $j=1,2,3$.

ТЕОрема 2.1. Каждый инвариантный дифференциалъный оператор порядка 1 с точностъю до перестановок и дуализащий кратен одному из следующих операторов: при $(\lambda, \gamma, \tau)=(0,0,0)$ - оператору

$\varphi(d x)^{\lambda} \otimes \psi(d x)^{\gamma} \otimes \chi(d x)^{\tau} \mapsto\left(\epsilon_{1} \cdot d \varphi \cdot \psi \cdot \chi+\epsilon_{2} \cdot \varphi \cdot d \psi \cdot \chi+\epsilon_{3} \cdot \varphi \cdot \psi \cdot d \chi\right)(d x)^{\lambda+\gamma+\tau+1}$, nрu $\lambda \neq 0$ - onepamopy

$$
\begin{aligned}
\varphi(d x)^{\lambda} \otimes \psi(d x)^{\gamma} \otimes \chi(d x)^{\tau} \mapsto & \left(\epsilon_{1}\left\{\varphi(d x)^{\lambda}, \psi(d x)^{\gamma}\right\} \chi(d x)^{\tau}+\right. \\
& \left.+\epsilon_{2}\left\{\varphi(d x)^{\lambda}, \chi(d x)^{\tau}\right\} \psi(d x)^{\gamma}\right)(d x)^{\lambda+\gamma+\tau+1} .
\end{aligned}
$$

ТЕОРема 2.2. Каждый инвариантный дифференииалъный оператор порядка 2 с точностъю до перестановок и дуализащий кратен одному из следующих операторов: $п р и(\gamma, \lambda)=(0,0)$ - оператору

$$
\begin{aligned}
\varphi(d x)^{\lambda} \otimes \psi(d x)^{\gamma} \otimes \chi(d x)^{\tau} \mapsto & \epsilon_{1}\{\varphi, d \psi\} \chi(d x)^{\tau}+ \\
& +\epsilon_{2}\left\{\chi(d x)^{\tau}, d \varphi\right\} \psi+\epsilon_{3}\left\{d \psi, \chi(d x)^{\tau}\right\} \varphi
\end{aligned}
$$

в остальных случаях - оператору

$$
\begin{aligned}
\varphi(d x)^{\lambda} \otimes \psi(d x)^{\gamma} \otimes \chi(d x)^{\tau} \mapsto & \epsilon_{1}\left\{\left\{\varphi(d x)^{\lambda}, \psi(d x)^{\gamma}\right\}, \chi(d x)^{\tau}\right\}+ \\
& +\epsilon_{2}\left\{\left\{\varphi(d x)^{\lambda}, \chi(d x)^{\tau}\right\}, \psi(d x)^{\gamma}\right\} .
\end{aligned}
$$

2.2.2. Порядок 3. Следующие тернарные $\mathfrak{v e c t}(1)$-инвариантные дифференциальные операторы являются новыми, новыми являются и их дуализации и перестановки (здесь $\mu=\lambda+\gamma+\tau+3)$ :

$$
\begin{gathered}
\Delta_{\lambda, \gamma, \tau ; 3}: \mathcal{F}_{\lambda} \otimes \mathcal{F}_{\gamma} \otimes \mathcal{F}_{\tau} \rightarrow \mathcal{F}_{\mu}, \\
\varphi(d x)^{\lambda} \otimes \psi(d x)^{\gamma} \otimes \chi(d x)^{\tau} \mapsto \sum_{i+j+l=3} \alpha_{i, j, l} \varphi^{(i)} \psi^{(j)} \chi^{(l)}(d x)^{\mu},
\end{gathered}
$$

где коэффициенты имеют вид

$$
\begin{aligned}
& \alpha_{3,0,0}=\gamma \tau(\tau-\gamma)(1+\gamma+\tau), \\
& \alpha_{2,0,1}=-\gamma(1+\gamma+\tau)(\gamma+2 \lambda+(3 \gamma+3 \lambda+4) \tau+2), \\
& \alpha_{1,0,2}=\gamma(1+\gamma+\lambda)(\gamma+2 \tau+\lambda(3 \gamma+3 \tau+4)+2), \\
& \alpha_{0,0,3}=\gamma \lambda(\lambda-\gamma)(1+\gamma+\lambda), \\
& \alpha_{2,1,0}=\tau(1+\gamma+\tau)(2 \lambda+\tau+\gamma(3 \lambda+3 \tau+4)+2), \\
& \alpha_{1,1,1}=(\lambda-\gamma)(\gamma-\tau)(\lambda-\tau), \\
& \alpha_{0,1,2}=-\lambda(1+\gamma+\lambda)(\lambda+2 \tau+\gamma(3 \lambda+3 \tau+4)+2), \\
& \alpha_{1,2,0}=-\tau(1+\lambda+\tau)(2 \gamma+\tau+\lambda(3 \gamma+3 \tau+4)+2), \\
& \alpha_{0,2,1}=\lambda(1+\lambda+\tau)(2 \gamma+\lambda+(3 \gamma+3 \lambda+4) \tau+2), \\
& \alpha_{0,3,0}=\lambda \tau(\tau-\lambda)(1+\lambda+\tau) .
\end{aligned}
$$


Следующее предложение показывает, что оператор $\Delta_{\lambda, \gamma, \tau ; 3}$ обобщает оператор Фейгина-Фукса $\Delta_{\lambda, 3}$.

ПРЕДЛОЖЕНИЕ 2.1. Если $\lambda=\gamma=\tau$, mo

$$
\Delta_{\lambda, \gamma, \tau ; 3}=-\lambda(1+2 \lambda)^{2}(2+3 \lambda) \Delta_{\lambda, 3}
$$

если $1+\lambda+\gamma=0$, mo

$$
\Delta_{\lambda, \gamma, \tau ; 3}=(\tau-\lambda)(1+\lambda+\tau)\{d\{\cdot, \cdot\}, \cdot\} .
$$

Дуализации и перестановки приводят к (новым) аналогам этого равенства.

ТеОРема 2.3. Каждый тернарный инвариантный дифберенииальный оператор порядка 3 с точностью до перестановок и дуализаций кратен одному из следующих операторов: при $(\gamma, \lambda, \tau)=(0,0,0)$ - оператору

$$
\begin{aligned}
\varphi(d x)^{\lambda} \otimes \psi(d x)^{\gamma} \otimes \chi(d x)^{\tau} \mapsto \epsilon_{1}\{d \varphi, d \psi\} \chi+\epsilon_{2}\{d \chi, d \varphi\} \psi+ \\
+\epsilon_{3}\{d \psi, d \chi\} \varphi+\epsilon_{4} d \varphi \otimes d \psi \otimes d \chi
\end{aligned}
$$

nрu $\lambda=0 u \gamma^{2}+\tau^{2} \neq 0$ - onepamopy

$$
\begin{aligned}
\varphi(d x)^{\lambda} \otimes \psi(d x)^{\gamma} \otimes \chi(d x)^{\tau} \mapsto \epsilon_{1}\left\{\left\{d \varphi, \psi(d x)^{\gamma}\right\}, \chi(d x)^{\tau}\right\}+ \\
+\epsilon_{2}\left\{\left\{d \varphi, \chi(d x)^{\tau}\right\}, \psi(d x)^{\gamma}\right\},
\end{aligned}
$$

nрu $(\gamma, \lambda, \tau)=(-2 / 3,-2 / 3,-2 / 3)$ - onepamopy

$$
\begin{aligned}
\varphi(d x)^{\lambda} & \otimes \psi(d x)^{\gamma} \otimes \chi(d x)^{\tau} \mapsto \epsilon_{1} \mathrm{Gz}\left(\varphi(d x)^{-2 / 3}, \psi(d x)^{-2 / 3}\right) \chi(d x)^{-2 / 3}+ \\
& +\epsilon_{2} \mathrm{Gz}\left(\chi(d x)^{-2 / 3}, \varphi(d x)^{-2 / 3}\right) \psi(d x)^{-2 / 3}+ \\
& +\epsilon_{3} \mathrm{Gz}\left(\psi(d x)^{-2 / 3}, \chi(d x)^{-2 / 3}\right) \varphi(d x)^{-2 / 3}
\end{aligned}
$$

nрu $(\gamma, \lambda, \tau)=(-1 / 2,-1 / 2,-1 / 2)$ - onepamopy

$$
\begin{aligned}
\varphi(d x)^{\lambda} & \otimes \psi(d x)^{\gamma} \otimes \chi(d x)^{\tau} \mapsto \epsilon_{1}\left\{d\left\{\varphi(d x)^{-1 / 2}, \psi(d x)^{-1 / 2}\right\}, \chi(d x)^{-1 / 2}\right\}+ \\
+ & \epsilon_{2}\left\{d\left\{\chi(d x)^{-1 / 2}, \varphi(d x)^{-1 / 2}\right\}, \psi(d x)^{-1 / 2}\right\}+ \\
+ & \epsilon_{3}\left\{d\left\{\psi(d x)^{-1 / 2}, \chi(d x)^{-1 / 2}\right\}, \varphi(d x)^{-1 / 2}\right\},
\end{aligned}
$$

npu $1+\lambda+\tau=0 u \gamma=\lambda \neq \tau-$ onepamopy

$$
\begin{gathered}
\varphi(d x)^{\lambda} \otimes \psi(d x)^{\gamma} \otimes \chi(d x)^{\tau} \mapsto \epsilon_{1}\left\{d\left\{\varphi(d x)^{\lambda}, \chi(d x)^{\tau}\right\}, \psi(d x)^{\gamma}\right\}+ \\
+\epsilon_{2}\left\{d\left\{\psi(d x)^{\gamma}, \chi(d x)^{\tau}\right\}, \varphi(d x)^{\lambda}\right\},
\end{gathered}
$$

в остальных случаях - оператору

$$
\varphi(d x)^{\lambda} \otimes \psi(d x)^{\gamma} \otimes \chi(d x)^{\tau} \mapsto \Delta_{\lambda, \gamma, \tau ; 3}\left(\varphi(d x)^{\lambda}, \psi(d x)^{\gamma}, \chi(d x)^{\tau}\right) .
$$


ДокАЗАТЕЛЬСтво. $\mathfrak{v e c t}(1)$-инвариантность дифференциального оператора

$$
A=\sum_{i+j+l=3} \alpha_{i, j, l} \varphi^{(i)} \psi^{(j)} \chi^{(l)}(d x)^{\mu}
$$

эквивалентна инвариантности по отношению ровно к двум полям, порождающим $\mathfrak{v e c t}(1)$, а именно $d / d x$ и $x^{3} \cdot d / d x$, и эквивалентна системе

$$
\begin{aligned}
& \alpha_{i, j, l}\left(\lambda\left(\begin{array}{c}
i \\
r-1
\end{array}\right)+\left(\begin{array}{c}
i \\
r
\end{array}\right)\right)+\alpha_{i-r+1, j+r-1, l}\left(\gamma\left(\begin{array}{c}
j+r-1 \\
r-1
\end{array}\right)+\left(\begin{array}{c}
j+r-1 \\
r
\end{array}\right)\right)+ \\
&+\alpha_{i-r+1, j, l+r-1}\left(\tau\left(\begin{array}{c}
l+r-1 \\
r-1
\end{array}\right)+\left(\begin{array}{c}
l+r-1 \\
r
\end{array}\right)\right)=0,
\end{aligned}
$$

где $i+j+l=3, r=2,3$.

План действий таков: найдем все решения системы (2.1) со всеми возможными значениями параметров $\lambda, \gamma$ и $\tau$. Пусть пространство решений $m$-мерно. Попытаемся построить $m$ линейно независимых операторов, выражающихся в терминах дифференциала де Рама, скобки Пуассона, оператора Грозмана или антисимметричных операторов Фейгина-Фукса: это возможно только для специальных значений $\lambda, \gamma$ и $\tau$. Если это невозможно (мы проверяем все возможные комбинации внешнего дифференциала и билинейных операторов), или если пространство, порожденное такими операторами, имеет размерность меньше $m$, то найденный оператор $A$ является новым. Возможны следующие значения $\lambda, \gamma, \tau$.

1. Случай $\lambda=\gamma=\tau=0$. В этом случае приведенная выше система допускает следующие решения:

$$
\begin{gathered}
\alpha_{0,0,3}=\alpha_{3,0,0}=\alpha_{0,3,0}=0, \quad \alpha_{0,2,1}=-\alpha_{0,1,2}, \\
\alpha_{2,0,1}=-\alpha_{1,0,2}, \quad \alpha_{2,1,0}=-\alpha_{1,2,0} .
\end{gathered}
$$

При этом мы хотим получить семейство операторов, зависящее от $\alpha_{1,2,0}, \alpha_{1,0,2}, \alpha_{1,1,1}$ и $\alpha_{0,1,2}$. С другой стороны, следующие операторы инвариантны и не могут быть кратными друг другу:

$$
\{d \varphi, d \psi\} \chi, \quad\{d \chi, d \varphi\} \psi, \quad\{d \psi, d \chi\} \varphi, \quad d \varphi \otimes d \psi \otimes d \chi
$$

Поэтому $A$ является линейной комбинацией этих инвариантных операторов.

2. Случай $\lambda=\gamma=0$ и $\tau \neq 0$ (случаи $\lambda=\tau=0, \gamma \neq 0$ и $\gamma=\tau=0, \lambda \neq 0$ получаются перестановками; случай $\tau=-2$ следует отбросить, поскольку он дуален случаю 1). Опуская подробности, заметим, что система (2.1) имеет решения, зависящие от двух параметров. Это означает, что $A$ есть линейная комбинация инвариантных операторов $\left\{d \varphi, \chi(d x)^{\tau}\right\} d \psi$ и $\left\{d \psi, \chi(d x)^{\tau}\right\} d \varphi$.

3. Случай $\lambda=0$ и $\gamma, \tau \neq 0$ аналогичен случаю 2 .

4. Случай $\lambda, \gamma, \tau \neq 0$ также аналогичен случаю 2.

ЗАмечАниЕ. Читатель, возможно, недоумевает, почему в нашем списке не появились операторы $\Delta_{\lambda, 3}$ при $\lambda=-1 / 2,-2 / 3,0$. Но в нашей классификации некоторые операторы обобщают операторы $\Delta_{\lambda, 3}$ для специальных значений $\epsilon_{j}$. Например, $\Delta_{-2 / 3,3}(\varphi, \psi, \chi)$ совпадает с оператором $\epsilon_{1} \mathrm{Gz}(\varphi, \psi) \chi+\epsilon_{2} \mathrm{Gz}(\chi, \varphi) \psi+\epsilon_{3} \mathrm{Gz}(\psi, \chi) \varphi$ при $\epsilon_{1}=\epsilon_{2}=\epsilon_{3}$. 
2.2.3. Порядок 4. Следующий тернарный инвариантный дифференциальный оператор является новым, новыми являются его дуализации и перестановки (здесь $\mu=\tau+5 / 2$ и $\tau \neq-3 / 4)$ :

$$
\begin{gathered}
\Xi: \mathcal{F}_{-\tau-3 / 2} \otimes \mathcal{F}_{\tau} \otimes \mathcal{F}_{\tau} \rightarrow \mathcal{F}_{\mu}, \\
\varphi(d x)^{-\tau-3 / 2} \otimes \psi(d x)^{\tau} \otimes \chi(d x)^{\tau} \mapsto \sum_{i+j+l=4} \alpha_{i, j, l} \varphi^{(i)} \psi^{(j)} \chi^{(l)}(d x)^{\mu},
\end{gathered}
$$

где коэффициенты равны

$$
\begin{aligned}
& \alpha_{4,0,0}=0, \quad \alpha_{3,0,1}=\frac{8}{3} \tau(2+3 \tau), \quad \alpha_{2,0,2}=4 \tau(2+3 \tau), \\
& \alpha_{1,0,3}=\frac{2}{3} \tau(13+12 \tau), \quad \alpha_{0,0,4}=\tau(3+2 \tau), \quad \alpha_{3,1,0}=-\frac{8}{3} \tau(2+3 \tau), \\
& \alpha_{2,1,1}=0, \quad \alpha_{1,1,2}=-10(1+\tau), \quad \alpha_{0,1,3}=-\frac{5}{3}(3+2 \tau), \\
& \alpha_{2,2,0}=-4 \tau(2+3 \tau), \quad \alpha_{1,2,1}=10(1+\tau), \quad \alpha_{0,2,2}=0, \\
& \alpha_{1,3,0}=-\frac{2}{3} \tau(13+12 \tau), \quad \alpha_{0,3,1}=\frac{5}{3}(3+2 \tau), \quad \alpha_{0,4,0}=-\tau(3+2 \tau) .
\end{aligned}
$$

При $\tau=-3 / 4$ имеется двухпараметрическое семейство инвариантных операторов, задающееся следующими коэффициентами:

$$
\begin{aligned}
& \alpha_{4,0,0}=t, \quad \alpha_{3,0,1}=\frac{4}{9}(s+5 t), \quad \alpha_{2,0,2}=\frac{2}{3} s, \\
& \alpha_{1,0,3}=-\frac{4}{9}(4 s+5 t), \quad \alpha_{0,0,4}=-s-t, \quad \alpha_{3,1,0}=\frac{4}{9}(4 t-s), \\
& \alpha_{2,1,1}=\frac{20}{9} t, \quad \alpha_{1,1,2}=-\frac{10}{9}(s+t), \quad \alpha_{0,1,3}=-\frac{4}{9}(5 s+4 t), \\
& \alpha_{2,2,0}=-\frac{2}{3}(s+t), \quad \alpha_{1,2,1}=\frac{20}{9} s, \quad \alpha_{0,2,2}=\frac{2}{3} t, \\
& \alpha_{1,3,0}=\frac{4}{9}(4 s-t), \quad \alpha_{0,3,1}=\frac{4}{9}(5 s+t), \quad \alpha_{0,4,0}=s .
\end{aligned}
$$

ТЕОРема 2.4. Каждый инвариантный дифференциалъный оператор порядка 4 с точностью до перестановок и дуализачий является кратным следующим операторам: при $(\gamma, \lambda)=(0,0)$ - оператору

$$
\varphi(d x)^{\lambda} \otimes \psi(d x)^{\gamma} \otimes \chi(d x)^{\tau} \mapsto \epsilon_{1}\left\{\{d \varphi, d \psi\}, \chi(d x)^{\tau}\right\}+\epsilon_{2}\left\{\left\{\chi(d x)^{\tau}, d \varphi\right\}, d \psi\right\},
$$

nрu $(\lambda, \gamma, \tau)=(-2,0,-2)-$ onepamopy

$$
\begin{aligned}
\varphi(d x)^{\lambda} \otimes \psi(d x)^{\gamma} \otimes \chi(d x)^{\tau} \mapsto & \epsilon_{1}\left\{d\left\{d \psi, \varphi(d x)^{-2}\right\}, \chi(d x)^{-2}\right\}+ \\
& +\epsilon_{2}\left\{d\left\{d \psi, \chi(d x)^{-2}\right\}, \varphi(d x)^{-2}\right\},
\end{aligned}
$$

nрu $\gamma=0, \lambda, \tau \neq 0$ - onepamopy

$$
\varphi(d x)^{\lambda} \otimes \psi(d x)^{\gamma} \otimes \chi(d x)^{\tau} \mapsto \Delta_{\lambda, \gamma, \tau ; 3}\left(\varphi(d x)^{\lambda}, d \psi, \chi(d x)^{\tau}\right),
$$


nри $(\lambda, \gamma)=(-2 / 3,-2 / 3)$ u $\tau \neq-1$ - onepamopy

$$
\varphi(d x)^{\lambda} \otimes \psi(d x)^{\gamma} \otimes \chi(d x)^{\tau} \mapsto\left\{\mathrm{Gz}\left(\varphi(d x)^{-2 / 3}, \psi(d x)^{-2 / 3}\right), \chi(d x)^{\tau}\right\},
$$

nри $(\lambda, \gamma, \tau)=(-1,-2 / 3,-2 / 3)-$ onepamopy

$$
\begin{aligned}
\varphi(d x)^{\lambda} \otimes \psi(d x)^{\gamma} \otimes \chi(d x)^{\tau} \mapsto & \epsilon_{1} \mathrm{Gz}\left(\left\{\varphi(d x)^{-1}, \psi(d x)^{-2 / 3}\right\}, \chi(d x)^{-2 / 3}\right)+ \\
& +\epsilon_{2} \mathrm{Gz}\left(\left\{\varphi(d x)^{-1}, \chi(d x)^{-2 / 3}\right\}, \psi(d x)^{-2 / 3}\right),
\end{aligned}
$$

при $(\lambda, \gamma, \tau)=(-3 / 2,-\tau, \tau)$ u $\tau \neq-3 / 2,-2 / 3,0,-3 / 4$ - onepamopy

$$
\varphi(d x)^{\lambda} \otimes \psi(d x)^{\gamma} \otimes \chi(d x)^{\tau} \mapsto \Xi\left(\varphi(d x)^{\lambda}, \psi(d x)^{\gamma}, \chi(d x)^{\tau}\right),
$$

nрu $(\lambda, \gamma, \tau)=(-3 / 4,-3 / 4,-3 / 4)$ - onepamopy

$$
\varphi(d x)^{\lambda} \otimes \psi(d x)^{\gamma} \otimes \chi(d x)^{\tau} \mapsto \Xi_{s, t}\left(\varphi(d x)^{\lambda}, \psi(d x)^{\gamma}, \chi(d x)^{\tau}\right) .
$$

2.2.4. Порядок 5. Следующий тернарный инвариантный дифференциальный оператор порядка 5 является новым, новыми являются его дуализации и перестановки (за исключением тех случаев, когда они совпадают):

$$
\begin{aligned}
\Gamma: \mathcal{F}_{-2 / 3} & \otimes \mathcal{F}_{-2 / 3} \otimes \mathcal{F}_{-4 / 3} \rightarrow \mathcal{F}_{7 / 3}, \quad \varphi(d x)^{-2 / 3} \otimes \psi(d x)^{-2 / 3} \otimes \chi(d x)^{-4 / 3} \mapsto \\
& \mapsto \sum_{i+j+l=5} \alpha_{i, j, l} \varphi^{(i)} \psi^{(j)} \chi^{(l)}(d x)^{7 / 3},
\end{aligned}
$$

где константы равны

$$
\begin{aligned}
& \alpha_{5,0,0}=-\frac{2}{5}, \quad \alpha_{4,0,1}=-\frac{17}{10}, \quad \alpha_{3,0,2}=-\frac{5}{2}, \quad \alpha_{2,0,3}=-1, \quad \alpha_{1,0,4}=1, \\
& \alpha_{0,0,5}=\frac{2}{5}, \quad \alpha_{4,1,0}=-\frac{3}{5}, \quad \alpha_{3,1,1}=-\frac{9}{4}, \quad \alpha_{2,1,2}=-\frac{9}{4}, \quad \alpha_{1,1,3}=\frac{3}{2}, \\
& \alpha_{0,1,4}=1, \quad \alpha_{3,2,0}=3, \quad \alpha_{2,2,1}=\frac{9}{2}, \quad \alpha_{1,2,2}=-\frac{9}{4}, \quad \alpha_{0,2,3}=-1, \\
& \alpha_{2,3,0}=3, \quad \alpha_{1,3,1}=-\frac{9}{4}, \quad \alpha_{0,3,2}=-\frac{5}{2}, \quad \alpha_{1,4,0}=-\frac{3}{5}, \quad \alpha_{0,4,1}=-\frac{17}{10}, \\
& \alpha_{0,5,0}=-\frac{2}{5} \text {. }
\end{aligned}
$$

Теорема 2.5. Каждый инвариантный дифференциалъный оператор порядка 5 с точностью до перестановок и дуализаций является кратным следующим операторам: при $(\lambda, \gamma, \tau)=(0,0,0)$ - оператору

$$
\varphi(d x)^{\lambda} \otimes \psi(d x)^{\gamma} \otimes \chi(d x)^{\tau} \mapsto \epsilon_{1}\{\{d \varphi, d \psi\}, d \chi\}+\epsilon_{2}\{\{d \chi, d \varphi\}, d \psi\},
$$

nрu $(\lambda, \gamma, \tau)=(0,0,-2)$ - onepamopy

$$
\begin{aligned}
\varphi(d x)^{\lambda} \otimes \psi(d x)^{\gamma} \otimes \chi(d x)^{\tau} \mapsto & \epsilon_{1}\left\{d\left\{d \varphi, \chi(d x)^{-2}\right\}, d \psi\right\}+ \\
& +\epsilon_{2}\left\{d\left\{d \psi, \chi(d x)^{-2}\right\}, d \varphi\right\}
\end{aligned}
$$


nрu $(\lambda, \gamma)=(0,0) u \tau \neq-4,-2,0$ - onepamopy

$$
\varphi(d x)^{\lambda} \otimes \psi(d x)^{\gamma} \otimes \chi(d x)^{\tau} \mapsto \Delta_{\lambda, \gamma, \tau ; 3}\left(d \varphi, d \psi, \chi(d x)^{\tau}\right),
$$

nрu $(\lambda, \gamma, \tau)=(-2 / 3,-2 / 3,0)$ - onepamopy

$$
\varphi(d x)^{\lambda} \otimes \psi(d x)^{\gamma} \otimes \chi(d x)^{\tau} \mapsto\left\{\mathrm{Gz}\left(\varphi(d x)^{-2 / 3}, \psi(d x)^{-2 / 3}\right), d \chi\right\},
$$

nрu $(\lambda, \gamma, \tau)=(-5 / 2,0,1)$ - onepamopy

$$
\varphi(d x)^{\lambda} \otimes \psi(d x)^{\gamma} \otimes \chi(d x)^{\tau} \mapsto \Xi\left(\varphi(d x)^{-5 / 2}, d \psi, \chi d x\right),
$$

nрu $(\lambda, \gamma, \tau)=(-2 / 3,-2 / 3,-4 / 3)$ - onepamopy

$$
\varphi(d x)^{\lambda} \otimes \psi(d x)^{\gamma} \otimes \chi(d x)^{\tau} \mapsto \Gamma\left(\varphi(d x)^{\lambda}, \psi(d x)^{\gamma}, \chi(d x)^{\tau}\right),
$$

при $\lambda=\gamma=\tau=-(9 \pm \sqrt{21}) / 12$ - onepamopy

$$
\varphi(d x)^{\lambda} \otimes \psi(d x)^{\gamma} \otimes \chi(d x)^{\tau} \mapsto \Theta_{ \pm}\left(\varphi(d x)^{\lambda}, \psi(d x)^{\gamma}, \chi(d x)^{\tau}\right) .
$$

\subsection{5. Порядок 6 .}

ТЕОРема 2.6. Каждый инвариантный дифференциалъный оператор порядка 6 является с точностью до перестановок и дуализаций кратным следующим операторам: при $(\lambda, \gamma, \tau)=(0,0,-2)$ - оператору

$$
\varphi(d x)^{\lambda} \otimes \psi(d x)^{\gamma} \otimes \chi(d x)^{\tau} \mapsto \Delta_{1,3}(d \varphi, d \psi, d \chi),
$$

nрu $(\lambda, \gamma, \tau)=(0,0,-5 / 2)$ - onepamopy

$$
\varphi(d x)^{\lambda} \otimes \psi(d x)^{\gamma} \otimes \chi(d x)^{\tau} \mapsto \Xi\left(d \varphi, d \psi, \chi(d x)^{-5 / 2}\right),
$$

nрu $(\lambda, \gamma, \tau)=(-5 / 4,-5 / 4,-5 / 4)$ - onepamopy

$$
\varphi(d x)^{\lambda} \otimes \psi(d x)^{\gamma} \otimes \chi(d x)^{\tau} \mapsto \Upsilon\left(\varphi(d x)^{-5 / 4}, \psi(d x)^{-5 / 4}, \chi(d x)^{-5 / 4}\right) .
$$

\subsection{6. Порядок больше 6 .}

Теорема 2.7. Не существует тернарных $\mathfrak{v e c t}(1)$-инвариантных дифференииальных операторов порядка больше 6.

ДокАзАтЕльство. Утверждение теоремы можно проверить прямым вычислением для операторов порядка $k=7$. Чтобы доказать результат для любого порядка $k>7$, будем действовать следующим образом.

Шаг 1. Случай $\lambda \gamma \tau \neq 0$. В этом случае инвариантность относительно векторного поля $x^{k+1} \cdot d / d x$, где $k$ - порядок оператора, эквивалентна системе

$$
\begin{aligned}
& \alpha_{i, j, l}\left(\lambda\left(\begin{array}{c}
i \\
r-1
\end{array}\right)+\left(\begin{array}{l}
i \\
r
\end{array}\right)\right)+\alpha_{i-r+1, j+r-1, l}\left(\gamma\left(\begin{array}{c}
j+r-1 \\
r-1
\end{array}\right)+\left(\begin{array}{c}
j+r-1 \\
r
\end{array}\right)\right)+ \\
& \quad+\alpha_{i-r+1, j, l+r-1}\left(\tau\left(\begin{array}{c}
l+r-1 \\
r-1
\end{array}\right)+\left(\begin{array}{c}
l+r-1 \\
r
\end{array}\right)\right)=0,
\end{aligned}
$$


где $i+j+l=k$ и $r=2, \ldots, k+1$. Сначала докажем, что для решений системы $(2.4)$ имеют место следующие равенства:

$$
\begin{aligned}
a_{k, 0,0} & =a_{0, k, 0}=a_{0,0, k}=a_{k-1, i, j}=a_{i, k-1, j}= \\
& =a_{i, j, k-1}=a_{k-2, i, j}=a_{i, k-2, j}=a_{i, j, k-2}=0 .
\end{aligned}
$$

Для этого рассмотрим подсистему системы (2.4), коэффициенты которой такие же, как в (2.5) при $r=k+1, k, k-1$ и $r=3,2$. Тогда получим линейную систему с матрицей размера $23 \times 18$ (данная матрица слишком громоздка, чтобы здесь ее приводить). При $\lambda \gamma \tau \neq 0$ эта матрица имеет ранг 18, а потому все решения нулевые, как в (2.5).

Пусть

$$
\alpha_{k-t, i, t-i}=\alpha_{i, k-t, t-i}=\alpha_{i, t-i, k-t}=0 \quad \text { при всех } \quad t, i=0, \ldots, n .
$$

Проведем индукцию по $n$ с целью доказать, что оставшиеся коэффициенты равны нулю.

При $r=k-n$ возьмем $i=k-n-1$ и любое $j=s \neq 0, n+1$ в системе (2.4). Получим

$$
\lambda \alpha_{k-n-1, s, n+1-s}+\left(\gamma\left(\begin{array}{c}
s+k-n-1 \\
k-n-1
\end{array}\right)+\left(\begin{array}{c}
s+k-n-1 \\
k-n
\end{array}\right)\right) \alpha_{0, k-n-1+s, n+1-s}=0 .
$$

Предположение индукции означает, что $\alpha_{0, k-n-1+s, n+1-s}=0$, поэтому

$$
\alpha_{k-n-1, s, n+1-s}=0 \quad \text { при всех } s \neq 0, n+1 .
$$

Аналогично можно доказать, что $\alpha_{s, k-n-1, n+1-s}=\alpha_{s, n+1-s, k-n-1}=0$ при всех $s \neq 0, n+1$.

При $r=3$ возьмем $i=2$ и $j=n-1$ в системе (2.4). Получим

$$
\begin{aligned}
& \left(\gamma\left(\begin{array}{c}
n+1 \\
2
\end{array}\right)+(n+1)\right) \alpha_{0, n+1, k-n-1}=0 \\
& \left(\gamma\left(\begin{array}{c}
n+1 \\
2
\end{array}\right)+\left(\begin{array}{c}
n+1 \\
3
\end{array}\right)\right) \alpha_{k-n-1, n+1,0}=0 \\
& \left(\lambda\left(\begin{array}{c}
n+1 \\
2
\end{array}\right)+\left(\begin{array}{c}
n+1 \\
3
\end{array}\right)\right) \alpha_{n+1,0, k-n-1}=0 \\
& \left(\lambda\left(\begin{array}{c}
n+1 \\
2
\end{array}\right)+\left(\begin{array}{c}
n+1 \\
3
\end{array}\right)\right) \alpha_{n+1, k-n-1,0}=0 \\
& \left(\tau\left(\begin{array}{c}
n+1 \\
2
\end{array}\right)+\left(\begin{array}{c}
n+1 \\
3
\end{array}\right)\right) \alpha_{0, k-n-1, n+1}=0 \\
& \left(\tau\left(\begin{array}{c}
n+1 \\
2
\end{array}\right)+\left(\begin{array}{c}
n+1 \\
3
\end{array}\right)\right) \alpha_{k-n-1,0, n+1}=0
\end{aligned}
$$


При $r=2$ возьмем $i=1$ и $j=n$ в системе (2.4). Получим

$$
\begin{aligned}
& \left(\gamma(n+1)+\left(\begin{array}{c}
n+1 \\
2
\end{array}\right)\right) \alpha_{0, n+1, k-n-1}=0, \quad\left(\gamma(n+1)+\left(\begin{array}{c}
n+1 \\
2
\end{array}\right)\right) \alpha_{k-n-1, n+1,0}=0, \\
& \left(\lambda(n+1)+\left(\begin{array}{c}
n+1 \\
2
\end{array}\right)\right) \alpha_{n+1,0, k-n-1}=0, \quad\left(\lambda(n+1)+\left(\begin{array}{c}
n+1 \\
2
\end{array}\right)\right) \alpha_{n+1, k-n-1,0}=0, \\
& \left(\tau(n+1)+\left(\begin{array}{c}
n+1 \\
2
\end{array}\right)\right) \alpha_{0, k-n-1, n+1}=0, \quad\left(\tau(n+1)+\left(\begin{array}{c}
n+1 \\
2
\end{array}\right)\right) \alpha_{k-n-1,0, n+1}=0 .
\end{aligned}
$$

Из уравнений (2.7) и (2.8) следует, что

$$
\begin{aligned}
\alpha_{k-n-1,0, n+1} & =\alpha_{k-n-1, n+1,0}=\alpha_{0, k-n-1, n+1}= \\
& =\alpha_{n+1, k-n-1,0}=\alpha_{0, n+1, k-n-1}=\alpha_{n+1,0, k-n-1}=0 .
\end{aligned}
$$

Из вида уравнений (2.6) и (2.9) следует, что утверждение индукции верно при $n+1$, откуда и получаем искомый результат.

Шаг 2. Случай $\lambda \gamma \tau=0$. Мы приведем здесь доказательство только в случае, когда $\tau=0$ и $\lambda \gamma \neq 0$. Тогда оператор $A(\cdot, \cdot, 1)$ превращается в $\mathfrak{v e c t}(1)$-инвариантный бинарный оператор, поскольку $\tau=0$. Из классификации Грозмана следует, что $A(\cdot, \cdot, 1)$ - нулевой оператор, поскольку $k>7$. Поэтому $\alpha_{i, k-i, 0}=0$ при $i=0, \ldots, k$. Таким образом, $A=B(\cdot, \cdot, d)$, где $B$ - оператор порядка $k-1$, определенный на $\mathcal{F}_{\lambda} \otimes \mathcal{F}_{\gamma} \otimes \mathcal{F}_{1}$. При этом $\mathfrak{v e c t}(1)$-инвариантность оператора $A$ автоматически влечет $\mathfrak{v e c t}(1)$-инвариантность оператора $B$. В силу шага 1 оператор $B$ должен быть нулевым, как и $A$.

\section{3. ТЕРНАРНЫЕ ИНВАРИАНТНЫЕ ДИФФЕРЕНЦИАЛЬНЫЕ ОПЕРАТОРЫ НА МНОГОМЕРНЫХ МНОГООБРАЗИЯХ}

Теперь рассмотрим $M=\mathbb{R}^{n}$. Пусть $g$ обозначает обычную псевдориманову метрику на $\mathbb{R}^{n}$ сигнатуры $(p, q)$, где $p+q=n$. Конформные преобразования порождаются векторными полями ${ }^{1)}$

$$
\begin{aligned}
X_{i} & =\frac{\partial}{\partial x^{i}}, & X_{i j} & =x_{i} \frac{\partial}{\partial x^{j}}-x_{j} \frac{\partial}{\partial x^{i}}, \\
X_{0} & =x^{i} \frac{\partial}{\partial x^{i}}, & \overline{X_{i}} & =x_{j} x^{j} \frac{\partial}{\partial x^{i}}-2 x_{i} x^{j} \frac{\partial}{\partial x^{j}},
\end{aligned}
$$

где $\left(x^{1}, \ldots, x^{n}\right)$ - координаты на $\mathbb{R}^{n}$ и $x_{i}=g_{i}^{j} x^{j}$. Ниже предполагается, что конформная алгебра Ли $\mathfrak{o}(p+1, q+1)$ реализована операторами (3.1). Очевидно, что алгебра Ли, порожденная векторными полями $X_{i j}$, изоморфна $\mathfrak{o}(p, q)$. Поэтому имеем вложение алгебр Ли

$$
\mathfrak{o}(p, q) \subset \mathfrak{o}(p+1, q+1) \subset \mathfrak{v} \mathfrak{e c t}(n) .
$$

Сначала мы проведем классификацию всех тернарных $\mathfrak{o}(p+1, q+1)$-инвариантных дифференциальных операторов, а затем используем результат для классификации $\mathfrak{v e c t}(1)$-инвариантных операторов.

\footnotetext{
1) Мы используем соглашение о суммировании по повторяющимся индексам. Индексы поднимаются и опускаются метрикой $g$.
} 
3.1. Конформно-инвариантные операторы. Приведенный ниже метод принадлежит Овсиенко и Реду [23]. Отождествим тернарные дифференциальные операторы с их символами. Это отождествление позволяет изучать подалгебру $\mathfrak{o}(p, q)$ инвариантных многочленов

$$
I:=\mathbb{C}\left[x^{1}, \ldots, x^{n}, \xi_{1}, \ldots, \xi_{n}, \eta_{1}, \ldots, \eta_{n}, \zeta_{1}, \ldots, \zeta_{n}\right]^{\mathfrak{o}(p, q)},
$$

воспользовавшись теорией инвариантов Вейля [26]. Получаем, что у алгебры $I$ следующие образующие:

$$
\begin{aligned}
& R_{x x}=x^{i} x_{j}, \quad R_{x \xi}=x^{i} \xi_{i}, \quad R_{x \eta}=x^{i} \eta_{i}, \quad R_{x \zeta}=x^{i} \zeta_{i}, \quad R_{\xi \xi}=\xi^{i} \xi_{j}, \\
& R_{\xi \eta}=\xi^{i} \eta_{i}, \quad R_{\xi \zeta}=\xi^{i} \zeta_{i}, \quad R_{\eta \eta}=\eta^{i} \eta_{i}, \quad R_{\eta \zeta}=\eta^{i} \zeta_{i}, \quad R_{\zeta \zeta}=\zeta^{i} \zeta_{i} .
\end{aligned}
$$

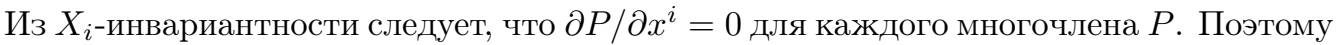
генераторы из списка (3.2), которые содержат $x$, следует отбросить. Таким образом, любой $\mathfrak{o}(p+1, q+1)$-инвариантный дифференциальный оператор должен иметь вид

$$
B=\sum_{a, b, c, d, e, f \geqslant 0} \alpha_{a b c d e f} R^{a, b, c, d, e, f}
$$

где $R^{a, b, c, d, e, f}=R_{\xi \xi}^{a} R_{\xi \eta}^{b} R_{\xi \zeta}^{c} R_{\eta \eta}^{d} R_{\eta \zeta}^{e} R_{\zeta \zeta}^{f}$.

ТеОРема 3.1. Для почти всех $\lambda, \gamma$ и существуют $\mathfrak{o}(p+1, q+1)$-инвариантные onepamopu

$$
B_{2 k}=\sum_{a+b+c+d+e+f=k} \alpha_{a b c d e f} R^{a, b, c, d, e, f}: \mathcal{F}_{\lambda} \otimes \mathcal{F}_{\gamma} \otimes \mathcal{F}_{\tau} \rightarrow \mathcal{F}_{\lambda+\gamma+\tau+2 k / n}
$$

где $k=0,1, \ldots$, а константы выражаются по индукции в соответствии с формулами

$$
\begin{aligned}
2(a+ & 1)(2(a+1)+n(2 \lambda-1)) \alpha_{a+1, b, c, d, e, f}- \\
& -(b+2)(b+1) \alpha_{a, b+2, c, d-1, e, f}-2(b+1)(c+1) \alpha_{a, b+1, c+1, d, e-1, f}+ \\
& +2(b+1)(b+e+2 d+n \gamma) \alpha_{a, b+1, c, d, e, f}-(c+2)(c+1) \alpha_{a, b, c+2, d, e, f-1}+ \\
& +2(c+1)(c+e+2 f+n \tau) \alpha_{a, b, c+1, d, e, f}=0, \\
2(d+ & 1)(2(d+1)+n(2 \gamma-1)) \alpha_{a, b, c, d+1, e, f}- \\
& -(b+2)(b+1) \alpha_{a-1, b+2, c, d, e, f}-2(b+1)(e+1) \alpha_{a, b+1, c-1, d, e+1, f}+ \\
& +2(b+1)(b+c+2 a+n \lambda) \alpha_{a, b+1, c, d, e, f}-(e+2)(e+1) \alpha_{a, b, c, d, e+2, f-1}+ \\
& +2(e+1)(c+e+2 f+n \tau) \alpha_{a, b, c, d, e+1, f}=0, \\
2(f+ & 1)(2(f+1)+n(2 \tau-1)) \alpha_{a, b, c, d, e, f+1}- \\
& -(e+2)(e+1) \alpha_{a, b, c, d-1, e+2, f}-2(e+1)(c+1) \alpha_{a, b-1, c+1, d, e+1, f}+ \\
& +2(e+1)(b+e+2 d+n \gamma) \alpha_{a, b, c, d, e+1, f}-(c+2)(c+1) \alpha_{a-1, b, c+2, d, e, f}+ \\
& +2(c+1)(c+b+2 a+n \lambda) \alpha_{a, b, c+1, d, e, f}=0 .
\end{aligned}
$$


ПримеР. При $k=1$ имеем (здесь $s, t$ и $u$ - параметры)

$$
\begin{aligned}
B_{2}= & n(\gamma s+\tau t)(2+n(2 \gamma-1))(2+n(2 \tau-1)) R_{\xi \xi}+ \\
& +n(\lambda s+\tau u)(2+n(2 \lambda-1))(2+n(2 \tau-1)) R_{\eta \eta}+ \\
& +n(\gamma u+\lambda t)(2+n(2 \lambda-1))(2+n(2 \gamma-1)) R_{\zeta \zeta}- \\
& -(2+n(2 \lambda-1))(2+n(2 \gamma-1))(2+n(2 \tau-1))\left(s R_{\xi \eta}+t R_{\xi \zeta}+u R_{\eta \zeta}\right) .
\end{aligned}
$$

ДоказАТЕЛЬство теоремы. Из $X_{0}$-инвариантности следует, что (здесь $L_{X}-$ производная Ли вдоль поля $X$ )

$$
L_{X_{0}} B=\sum_{a, b, c, d, e, f \geqslant 0}(n(\mu-\lambda-\gamma-\tau)-2(a+b+c+d+e+f)) c_{a, b, c, d, e, f} R^{a, b, c, d, e, f}=0 .
$$

Поэтому $n(\mu-\lambda-\gamma-\tau)-2(a+b+c+d+e+f)=0$. Таким образом, оператор $B_{2 k}$ является однородным (все его компоненты имеют одну и ту же степень). Для получения индукционных формул нам требуется следующее предложение (см., например, [27]).

ПреДЛОЖенИЕ 3.1. Действие оператора $\overline{X_{i}}$ имеет вид $L_{\overline{X_{i}}}^{\lambda, \gamma, \tau ; \mu}=L_{\overline{X_{i}}}^{\mu-\lambda-\gamma-\tau}-\xi_{i} T_{\xi}-\eta_{i} T_{\eta}-\zeta_{i} T_{\zeta}+2\left(\left(E_{\xi}+n \lambda\right) \partial_{\xi^{i}}+\left(E_{\eta}+n \gamma\right) \partial_{\eta^{i}}+\left(E_{\zeta}+n \tau\right) \partial_{\zeta^{i}}\right)$, где

$$
\begin{aligned}
L_{\overline{X_{i}}}^{\mu-\lambda-\gamma-\tau}= & x_{j} x^{j} \partial_{i}-2 x_{i} x^{j} \partial_{j}-2 n(\mu-\lambda-\gamma-\tau) x_{i}- \\
& -2\left(\left(\xi_{i} x_{j}-\xi_{j} x_{i}\right) \partial_{\xi_{j}}+\left(\eta_{i} x_{j}-\eta_{j} x_{i}\right) \partial_{\eta_{j}}+\left(\zeta_{i} x_{j}-\zeta_{j} x_{i}\right) \partial_{\zeta_{j}}\right)+ \\
& +2\left(\xi_{j} x^{j} \partial_{\xi^{i}}+\eta_{j} x^{j} \partial_{\eta^{i}}+\zeta_{j} x^{j} \partial_{\zeta^{i}}\right),
\end{aligned}
$$

$T_{\xi}=\partial_{\xi^{j}} \partial_{\xi_{j}}-$ cлeд, a $E_{\xi}=\xi_{j} \partial_{\xi_{j}}-$ onepamop Эйлера.

Из предложения 3.1 следует, что

$$
\begin{aligned}
L_{X}^{\lambda, \gamma, \tau ; \mu} & R^{a, b, c, d, e, f}=2(2 k-n(\mu-\lambda-\gamma-\tau)) R^{a, b, c, d, e, f} x_{i}+ \\
& +\left(2 a(2 a+n(2 \lambda-1)) R^{a-1, b, c, d, e, f}-b(b-1) R^{a, b-2, c, d+1, e, f}-\right. \\
& -2 b c R^{a, b-1, c-1, d, e+1, f}+2 b(b-1+e+2 d+n \gamma) R^{a, b-1, c, d, e, f}- \\
& \left.-c(c-1) R^{a, b, c-2, d, e, f+1}+2 c(c-1+e+2 f+n \tau) R^{a, b, c-1, d, e, f}\right) \xi_{i}+ \\
& +\left(2 d(2 d+n(2 \gamma-1)) R^{a, b, c, d-1, e, f}-b(b-1) R^{a+1, b-2, c, d, e, f}-\right. \\
& -2 b e R^{a, b-1, c+1, d, e-1, f}+2 b(b-1+c+2 a+n \lambda) R^{a, b-1, c, d, e, f}- \\
& \left.-e(e-1) R^{a, b, c, d, e-2, f+1}+2 e(e-1+c+2 f+n \tau) R^{a, b, c, d, e-1, f}\right) \eta_{i}+ \\
& +\left(2 f(2 f+n(2 \tau-1)) R^{a, b, c, d, e, f-1}-e(e-1) R^{a, b, c, d+1, e-2, f}-\right. \\
& -2 e c R^{a, b+1, c-1, d, e-1, f}+2 e(e-1+b+2 d+n \gamma) R^{a, b, c, d, e-1, f}- \\
& \left.-c(c-1) R^{a+1, b, c-2, d, e, f}+2 c(c-1+b+2 a+n \lambda) R^{a, b, c-1, d, e, f}\right) \zeta_{i} .
\end{aligned}
$$

Действуя теперь с помощью $L_{X}^{\lambda, \gamma, \tau ; \mu}$ на $B_{2 k}$, после перегруппировки слагаемых получаем рекуррентную систему линейных уравнений (3.5). Операторы $B_{2 k}$ зависят от некоторых параметров и могут обращаться в нуль при некоторых специальных значениях $\lambda, \gamma$ и $\tau$. 
3.2. $\mathfrak{v e c t}(n)$-инвариантные дифференциальные операторы. Поскольку каждый $\mathfrak{v e c t}(n)$-инвариантный оператор является также $\mathfrak{o}(p+1, q+1)$-инвариантным, достаточно проверить, являются ли $\mathfrak{v e c t}(n)$-инвариантными конформно-инвариантные операторы, перечисленные в теореме 3.1 .

ТЕОРема 3.2. Единственный $\mathfrak{v e t}(n)$-инвариантный тернарный дифберенииальный оператор является оператором умножения:

$$
\mathcal{F}_{\lambda} \otimes \mathcal{F}_{\gamma} \otimes \mathcal{F}_{\tau} \rightarrow \mathcal{F}_{\lambda+\gamma+\tau}, \quad \varphi|\operatorname{vol}|^{\lambda} \otimes \psi|\operatorname{vol}|^{\gamma} \otimes \chi|\operatorname{vol}|^{\tau} \mapsto \varphi \cdot \psi \cdot \chi|\operatorname{vol}|^{\lambda+\gamma+\tau} .
$$

ДокАЗАТЕЛЬство. Прямое вычисление показывает, что действие посредством $X \in \mathfrak{v e c t}(n)$ имеет вид (cp. с действием из предложения 3.1)

$$
\begin{aligned}
L_{X}^{\lambda, \gamma, \tau ; \mu}= & (\mu-\lambda-\gamma-\tau) \operatorname{Div}(X)-\left(\partial_{j} X^{m} \xi_{m} \partial_{\xi_{j}}+\partial_{j} X^{m} \eta_{m} \partial_{\eta_{j}}+\partial_{j} X^{m} \zeta_{m} \partial_{\zeta_{j}}\right)+ \\
& +\left(\text { производные более высоких порядков } \partial_{i_{1}} \ldots \partial_{i_{l}} X\right) .
\end{aligned}
$$

Поскольку каждый $\mathfrak{v e c t}(n)$-инвариантный оператор является $\mathfrak{o}(p+1, q+1)$-инвариантным, имеем (см. (3.4))

$L_{X}^{\lambda, \gamma, \tau}\left(B_{2 k}\right)=\sum_{a+b+c+d+e+f=k}(\mu-\lambda-\gamma-\tau) \alpha_{a, b, c, d, e, f} \operatorname{Div}(X) R^{a, b, c, d, e, f}+($ другие члены).

Таким образом, $(\mu-\lambda-\gamma-\tau) \alpha_{a, b, c, d, e, f}=0$. Поскольку $\mu-\lambda-\gamma-\tau=2 k / n$, получаем, что либо $k=0$, либо $\alpha_{a, b, c, d, e, f}=0$. Следовательно, если $B_{2 k}$ не является скалярным оператором (1.1), то он должен быть нулевым оператором.

Благодарности. Я благодарен Д. Лейтесу и В. Овсиенко за их интерес к настоящей работе и помощь.

\section{Список литературы}

[1] P. Grozman, D. Leites, I. Shchepochkina, "Invariant operators on supermanifolds and standard models", Multiple Facets of Quantization and Supersymmetry, eds. M. Olshanetsky, A. Vainstein, World Scientific, Singapore, 2002, 508-555.

[2] S. Hansoul, P. Lecomte, Internat. Math. Res. Notices, 2005, № 16, 981-1003.

[3] I. Kolàr, P. Michor, J. Slovák, Natural Operations in Differential Geometry, Springer, Berlin, 1993.

[4] А. А. Кириллов, "Инвариантные операторы над геометрическими величинами", Современные проблемы математики. Новейшие достижения, Итоги науки и техники, 16, ВИНИТИ, М., 1980, 3-29.

[5] А. Н. Рудаков, Изв. АН СССР. Сер. матем., 1974, № 4, 835-866.

[6] C. L. Terng, Amer. J. Math., 100:4 (1978), 775-828.

[7] П. Грозман, Функи. анализ и его прил., 14:2 (1980), 58-59; arXiv: math/0509562.

[8] Б. Л. Фейгин, Д. Б. Фукс, Функи. анализ и его прил., 16:2 (1982), 47-63.

[9] G. Bol, Abh. Math. Sem. Univ. Hamburg, 16:3-4 (1949), 1-28.

[10] P. Gordan, Vorlesungen über Invariantentheorie, Teubner, Leipzig, 1887.

[11] S. Bouarroudj, V. Ovsienko, Internat. Math. Res. Notices, 1998:1 (1998), 25-39.

[12] H. Cohen, Math. Ann., 217:3 (1977), 271-285.

[13] P. Cohen, Yu. Manin, D. Zagier, "Automorphic pseudodifferential operators", Algebraic Aspects of Integrable Systems, Progr. Nonlinear Differential Equations Appl., 26, eds. A. S. Fokas, I. M. Gelfand, Birkhäuser, Boston, MA, 1997, 17-47. 
[14] P. Olver, A. Jan Sanders, Adv. Appl. Math., 25:3 (2000), 252-283.

[15] R. A. Rankin, J. Indian Math. Soc., 20 (1956), 103-116.

[16] D. Zagier, Proc. Indian. Acad. Sci. Math. Sci., 104:1 (1994), 57-75.

[17] В. Овсиенко, С. Табачников, Проективная дифференииальная геометрия. Старое и новое: от производной Швари, до когомологий групп диффеоморфизмов, МЦНМО, M., 2008.

[18] S. Bouarroudj, Int. J. Geom. Methods Mod. Phys., 2:1 (2005), 23-40.

[19] F. Boniver, P. Mathonet, J. Math. Phys., 42:2 (2001), 582-589.

[20] M. G. Eastwood, J. W. Rice, Comm. Math. Phys., 109:2 (1987), 207-228.

[21] A. R. Gover, K. Hirachi, J. Amer. Math. Soc., 17:2 (2004), 389-405.

[22] H. P. Jakobsen, M. Vergne, J. Func. Anal., 24:1 (1977), 52-106.

[23] V. Ovsienko, P. Redou, Lett. Math. Phys., 63:1 (2003), 19-28.

[24] P. Mathonet, Lett. Math. Phys., 48:3 (1999), 251-261.

[25] S. Bouarroudj, J. Geom. Phys., 57:6 (2007), 1441-1456.

[26] Г. Вейль, Классические группь, их инварианты и представления, ИЛ, М., 1947.

[27] C. Duval, P. Lecomte, V. Ovsienko, Ann. Inst. Fourier, 49:6 (1999), 1999-2029.

Поступила в редакцию 22.05.2008 